\title{
Activation of free fatty acid receptor 1 improves hepatic steatosis through a p38-dependent pathway
}

\author{
Horng-Yih Ou, Hung-Tsung $\mathrm{Wu}^{1,2}$, Feng-Hwa $\mathrm{Lu}^{2}$, Yu-Chu Su${ }^{3}$, Hao-Chang Hung, \\ Jin-Shang $\mathrm{Wu}^{2}$, $\mathrm{Yi}$-Ching $\mathrm{Yang}^{2}$, Chao-Liang $\mathrm{Wu}^{3}$ and Chih-Jen Chang ${ }^{1,2}$ \\ Division of Endocrinology and Metabolism, Department of Internal Medicine, National Cheng Kung University \\ Hospital, 138, Sheng-Li Road, Tainan 70403, Taiwan \\ ${ }^{1}$ Research Center of Herbal Medicine, New Drugs, and Nutritional Supplements, National Cheng Kung University, \\ Tainan, Taiwan \\ ${ }^{2}$ Department of Family Medicine, National Cheng Kung University Hospital, 138, Sheng-Li Road, Tainan 70403, \\ Taiwan \\ ${ }^{3}$ College of Medicine, Institute of Basic Medical Sciences, National Cheng Kung University, Tainan, Taiwan
}

Correspondence should be addressed to C-J Chang Email changcj.ncku@gmail.com

\begin{abstract}
Hepatic steatosis is highly correlated with insulin resistance and diabetes. Although, it has been demonstrated that activation of free fatty acid receptor 1 (FFAR1) by agonists showed benefits for the improvement of diabetes, the effects of FFAR 1 agonists on hepatic steatosis were unknown. In this study, a high fat diet (HFD)-induced hepatic steatosis animal model was utilized to evaluate the effects of an FFAR1 agonist, GW9508, on hepatic lipid accumulation, and HepG2 hepatoma cells were also used to clarify the possible mechanisms. Administration of GW9508 significantly decreased the hepatic lipid accumulation with decreased expressions of lipogenesis-related proteins in HFD mice. Knockdown of hepatic Ffar1 by lentiviral vectors containing short hairpin RNA targeted to Ffar1 diminished the effect of GW9508 in HFD mice. In addition, GW9508 decreased oleic acid-induced lipid accumulation in HepG2 cells by decreases in the expression of lipogenesis-related proteins. Moreover, GW9508 downregulated the expression of sterol regulatory element-binding protein 1 (SREBP1) through a p38-dependent pathway, whereas knockdown of Ffar1 in HepG2 cells diminished the effect of GW9508 on the decrease in SREBP1. Considering all these results together, GW9508 exerts a therapeutic effect to improve hepatic steatosis through a p38-dependent pathway. Thus, investigation of chemicals that act on FFAR1 might be a new strategy for the treatment of hepatic steatosis.
\end{abstract}

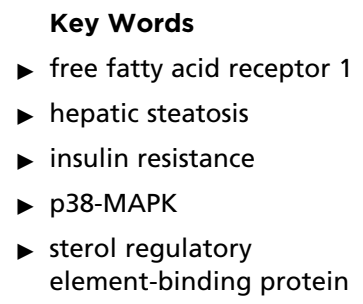

Journal of Molecular Endocrinology (2014) 53, 165-174

\section{Introduction}

Hepatic steatosis is characterized by the accumulation of triglyceride in the liver and is a common form of chronic liver disease. It is now considered to be a manifestation of metabolic syndrome and is highly correlated with type 2 diabetes and insulin resistance (Lewis \& Mohanty 2010). Although hepatic steatosis is thought to be benign, it is 
now clear that steatosis can evolve into more severe liver damage including steatohepatitis, fibrosis, cirrhosis, and hepatocellular carcinoma. However, there is still no effective treatment for hepatic steatosis, and life style modification remains the best therapeutic option (Ferre \& Foufelle 2010).

Free fatty acid receptor 1 (FFAR1), also named G-proteincoupled receptor 40 , is a seven-transmembrane-domain receptor, which couples with a G-protein $\alpha$-subunit of the Gq family to perform important functions in various physiological processes (Hara et al. 2011). It is detected primarily in pancreatic $\beta$-cells, and their physiological functions contribute to insulin secretion (Itoh et al. 2003). Activation of FFAR1 increases phospholipase C (PLC) activity through the Gq protein to facilitate insulin secretion (Feng et al. 2006). In addition, results from recent studies have indicated that FFAR1 is expressed in liver and regulates hepatic insulin sensitivity (Suh et al. 2008, Wu et al. 2012). Activation of FFAR1 decreased blood glucose, reduced plasma insulin and improved glucose intolerance and insulin resistance in high fat diet (HFD)-induced diabetic mice ( $\mathrm{Ou}$ et al. 2013). Assessment of the efficacy and safety of FFAR1 agonists has also been validated in patients with type 2 diabetes (Christiansen et al. 2013, Kaku et al. 2013). Although the therapeutic effects of FFAR1 on insulin resistance and diabetes are documented, the role of FFAR1 in hepatic steatosis is still unknown.

In this study, we used HFD-induced hepatic steatosis mice to evaluate the effects of the FFAR1 agonist GW9508 on lipid accumulation in liver. In addition, specific knockdown of hepatic FFAR1 in mice was achieved using lentiviral vectors containing short hairpin RNA (shRNA) targeted to FFAR1, to confirm the role of FFAR1 in GW9508-improved hepatic steatosis. Moreover, HepG2 cells were used to clarify the possible mechanisms of FFAR1-regulated hepatic lipogenesis.

\section{Materials and methods}

\section{Materials}

Chelethyrine and U73343 were purchased from SigmaAldrich. GW9805 and U73122 were purchased from Tocris Bioscience (Ellisville, MO, USA). Farnesyl thiosalicylic acid (FTS) and the WST-8 proliferation assay kit were purchased from Cayman Chemicals (Ann Arbor, MI, USA). The following primary antibodies were used: sterol regulatory element-binding protein 1 (SREBP1; Novus, Littleton, CO, USA), fatty acid synthase (FAS), and acetyl-CoA carboxylase (ACC) (Epitomics, Burlingame, CA, USA), RAS, phospho-PLC (pPLC), PLC, phospho-protein kinase C (pPKC), PKC, phospho-p38, and p38 (Cell Signaling Technology, Beverly, MA, USA), FFAR1 (Abcam, Cambridge, UK). Peroxisome proliferator-activated receptor alpha and gamma and actin (Sigma-Aldrich) were purchased from commercial companies.

\section{Animals}

The animal use protocol was reviewed and approved by Institutional Animal Care and Use Committee in National Cheng Kung University (permit no.101177). The animal experiments were conducted in accordance with the guide for the care and use of laboratory animals of the National Institutes of Health, as well as the guidelines of the Animal Welfare Act. All of the animal experiments were carried out under sodium pentobarbital anesthesia (SigmaAldrich), and all efforts were made to minimize suffering. C57BL/6J male mice were purchased from the animal center of National Cheng Kung University Medical College. Mice were housed in a temperature $\left(25 \pm 1^{\circ} \mathrm{C}\right)$ and humidity $(60 \pm 5 \%)$-controlled room and kept on a $12 \mathrm{~h}$ light: $12 \mathrm{~h}$ darkness cycle (light on at $0600 \mathrm{~h}$ ). C57BL/6J mice were fed with a HFD containing $34.9 \%$ fat (wt/wt) for 12 weeks (58Y1; TestDiet, Richmond, IN, USA) starting from 8 weeks of age. The mice were fed with a HFD diet containing GW9805 at the indicated doses for 30 days at the end of 3 months of HFD.

\section{Specific knockdown of hepatic FFAR1 by lentiviral vectors}

Specific knockdown of FFAR1 in the liver was performed using the methods employed in a previous study (Wu et al. 2013). Briefly, the lentiviral vectors with shRNA for human FFAR1 were purchased from OriGene Technologies (Rockville, MD, USA). Lentivirus production was performed as described previously (Wang et al. 2010). In brief, lentiviral vectors were transiently transfected with the packaging plasmid pSPAX2 and the VSV expression plasmid pMD2.G into 293T cells using calcium phosphate precipitation. After $48 \mathrm{~h}$, virus particles were collected from the supernatant and concentrated by ultracentrifugation. The relative infection unit (RIU) of lentivirus was determined using a standard method. In brief, $5 \times 10^{3}$ HepG2 cells were plated into each well of a 96-well plate. A series of diluted viruses were added and incubated for $4 \mathrm{~h}$. After removal of virus-containing media, $100 \mu \mathrm{l}$ growth media were added and incubated overnight. Then, the media were replaced with $100 \mu \mathrm{l}$

Published by Bioscientifica Ltd. 
puromycin ( $2 \mu \mathrm{g} / \mathrm{ml}$; Sigma-Aldrich)-containing media. After 48 h, cell viability was measured by the WST- 8 assay. RIU of lentivirus was determined by relative viability: $5 \times 10^{8}$ RIU $(100 \mu \mathrm{l})$ of letiviral vectors with scramble shRNA and shFFAR1 were used for injection into the portal veins of HFD-fed mice.

\section{Cell culture}

The HepG2 cell line was purchased from the Bioresource Collection and Research Center (Food Industry Research and Development Institute, Hsinchu, Taiwan). The cells were cultured at $37^{\circ} \mathrm{C}$ and $5 \% \mathrm{CO}_{2}$ in DMEM (Hyclone,
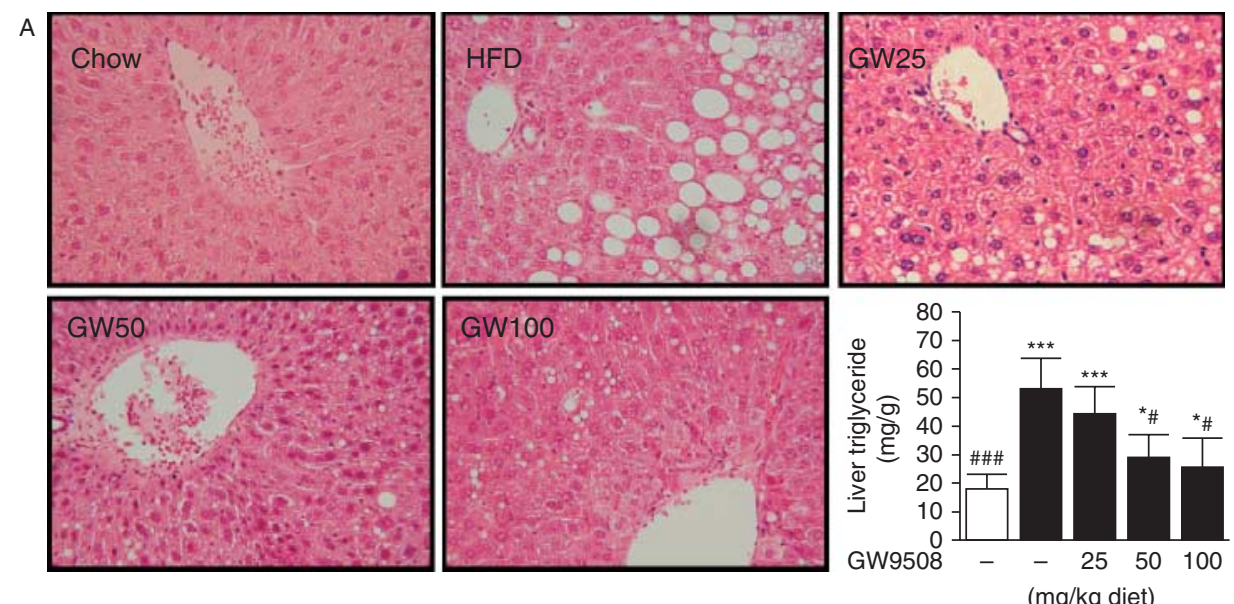

B
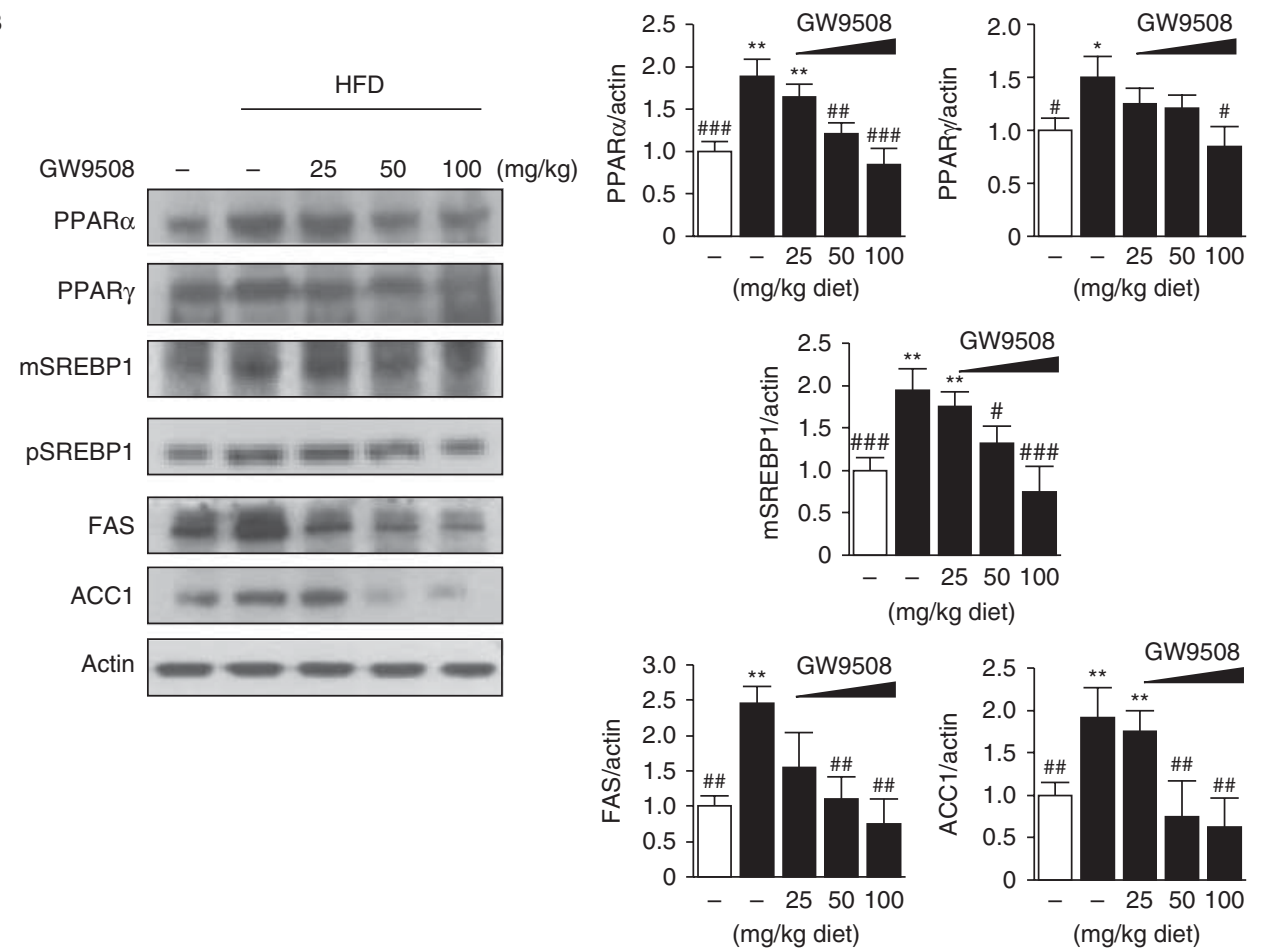

\section{Figure 1}

Administration of GW9508 improved high fat diet (HFD)-induced hepatic steatosis in mice. (A) Eight-week-old C57BL/6 mice were fed with HFD for 3-month to induce hepatic steatosis and then the mice were fed with the HFD diet supplied with different doses of GW9508. Liver samples were harvested after the treatment with GW9508 for 30 days. (B) Levels of expression of hepatic lipogenesis-related proteins in mice treated with GW9508 were determined by western blotting analysis. The quantifications

of protein expression were obtained from six to eight animals and expressed as mean \pm S.E.M. ${ }^{*} P<0.05 ; * * P<0.01$; and $* * * P<0.001$ as compared with the normal chow group. ${ }^{\#} P<0.05$; ${ }^{\# \#} P<0.01$; and ${ }^{\# \# \# ~} P<0.001$ as compared with the HFD group. GW100, $100 \mathrm{mg} / \mathrm{kg}$ diet GW9508; GW50, 50 mg/kg diet GW9508; GW25, 25 mg/kg diet GW9508; pSREBP, precursor SREBP; mSREBP, mature SREBP.

Published by Bioscientifica Ltd. 
South Logan, UT, USA) supplemented with $10 \%$ fetal bovine serum (Hyclone) and antibiotics (100 IU/ml penicillin and $100 \mathrm{mg} / \mathrm{ml}$ streptomycin) (Caisson Laboratories, Inc., North Logan, UT, USA). The cells were grown in six-well culture dishes at a density of $2 \times 10^{5}$ cells/well. For the induction of steatosis, the cells were starved in serum-free medium overnight. Oleic acid (OA; SigmaAldrich) was used for the treatment of HepG2 cells after conjugation with the appropriate concentration of BSA (Sigma-Aldrich). The final molar ratio of FFA:BSA was approximately $2: 1$, which was close to the ratio observed in human serum (Spector 1975). The control cells were treated with OA-free medium containing $0.5 \%$ BSA. Staining of intracellular neutral lipids was carried out using Oil Red O staining (Sigma-Aldrich).

\section{siRNA transfection}

HepG2 cells were transfected with duplexed RNA oligonucleotides (Stealth RNAi; Invitrogen) of human FFAR1 or scrambled siRNA (as a negative control) using Lipofectamine 2000 (Invitrogen) according to the manufacturer's instructions. The cells were treated with
GW9805 at 48-h posttransfection. The content of glycogen in cells was determined using a kit and following the manufacturer's protocol (BioVision, Mountain View, CA, USA).

\section{Western blot analysis}

The isolated cell fractions were obtained using commercially available kits according to the manufacturer's protocol (BioVision). Protein lysates $(30 \mu \mathrm{g})$ containing proteinase and phosphotase inhibitors cocktail (SigmaAldrich) of liver tissues or cells were separated using 10\% SDS-PAGE and transferred to a polyvinylidene difluoride membrane (Millipore, Billerica, MA, USA). After they had been blocked with $10 \%$ milk in TBS-T $(10 \mathrm{mM}$ Tris $(\mathrm{pH}$ 7.6), $150 \mathrm{mM} \mathrm{NaCl}$, and $0.05 \%$ Tween 20 ), the membranes were incubated with appropriate primary antibodies. After the membranes had been washed with TBS-T, the blots were incubated with a $1 / 5000$ dilution of HRP-conjugated secondary antibodies at $25^{\circ} \mathrm{C}$ for $1 \mathrm{~h}$. The relative signal intensity was quantified using ImageJ Software from W. Rasband (National Institutes of Health, Bethesda, MD, USA; http://rsb.info.nih.gov/ij/).

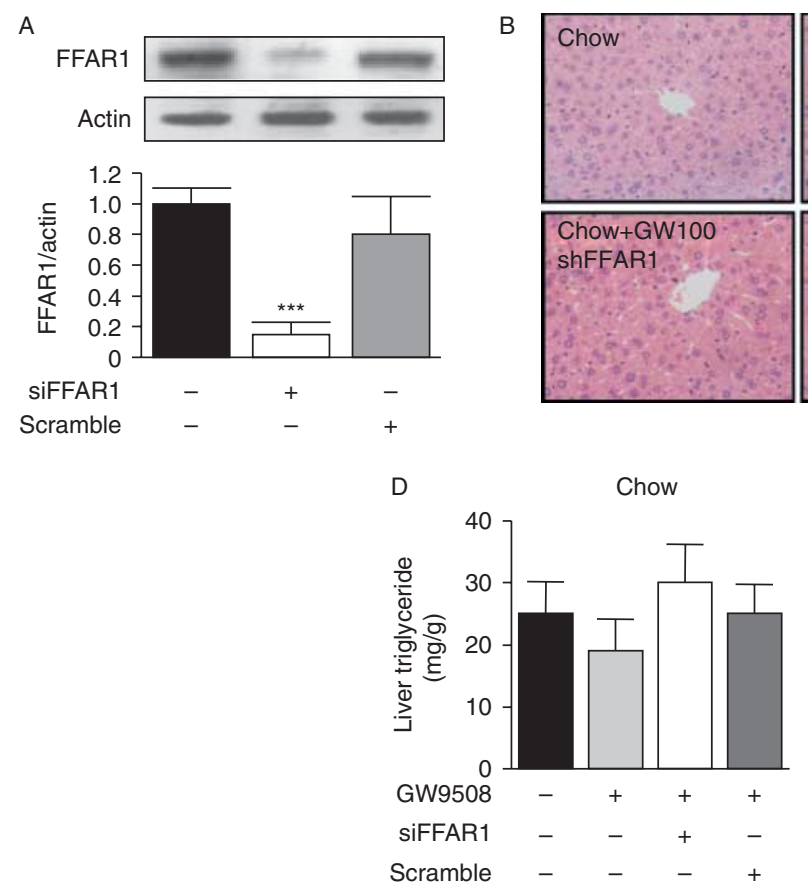

Figure 2

Knockdown of FFAR1 diminished the effect of GW9508 on hepatic steatosis. Lentiviral vector containing shRNA targeted to FFAR1 or scramble shRNA were delivered to high fat diet-fed mice (HFD) by portal vein injection. After the delivery of the lentiviral vectors for 2 weeks, the efficiency to FFAR1 knockdown was determined using western blotting analysis (A). The transfected chow-fed (B) or HFD mice (C) were then fed
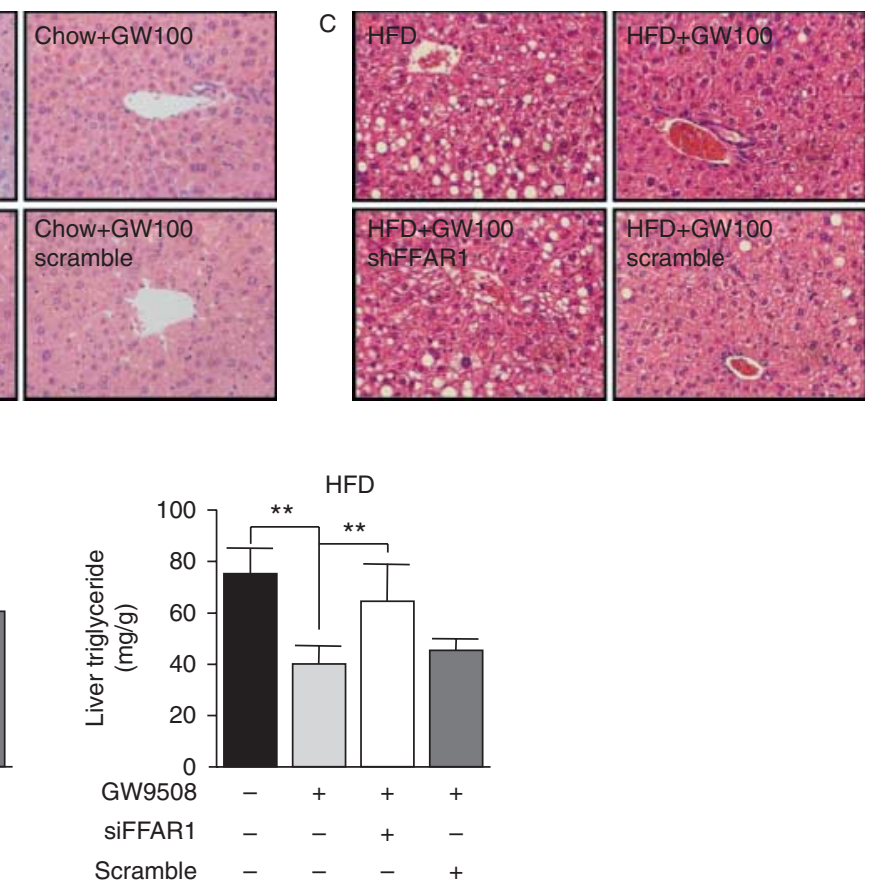

with the diets containing GW9508 (GW100, $100 \mathrm{mg} / \mathrm{kg}$ diet GW9508) for 30 days to evaluate the development of hepatic steatosis by H\&E staining and liver triglyceride contents (D). The data were obtained from six to eight animals and expressed as mean \pm S.E.M. ${ }^{*} * P<0.01$ and $* * * P<0.001$ as compared with the control group

Published by Bioscientifica Ltd. 


\section{Statistical analyses}

Student's two-tailed unpaired t-test or one-way ANOVA followed by Dunnet's post-hoc test were used for comparison of the variables among groups, and the data were expressed as mean \pm s.e.m. A $P$ value $<0.05$ was considered as statistically significant.

\section{Results}

Administration of GW9508, a FFAR1 agonist, improved HFD-induced hepatic steatosis in mice and knockdown of hepatic FFAR1 diminished the effect of GW9508 on hepatic steatosis

Administration of GW9508 for 30 days in HFD mice decreased hepatic triglyceride contents and improved hepatic steatosis (Fig. 1A). Levels of expression of hepatic lipogenesis-related protein were increased in HFD mice, whereas administration of GW9508 reversed these effects (Fig. 1B). To confirm the role of FFAR1 in the GW9508-improved hepatic steatosis, specific hepatic knockdown of FFAR1 in mice was achieved following the portal vein delivery of lentiviral vectors containing shRNA targeted to FFAR1 (Fig. 2A). Knockdown of hepatic FFAR1 expression with GW9508 showed no significant effects on hepatic lipid accumulation in chow-fed mice (Fig. 2C and D); however, knockdown of hepatic FFAR1 expression reversed the effect of GW9508 on hepatic steatosis, implying that FFAR1-mediated GW9508-improved hepatic steatosis (Fig. 2C and D). The body weight (Fig. 3A) and daily food intake (Fig. 3B) of each group of the mice showed no significant changes. Consistent with these results, knockdown of hepatic FFAR1 expression also reversed the effect of GW9508 on expression of lipogenesis-related proteins (Fig. 3C).

\section{Activation of FFAR 1 by GW9508 decreased OA-induced lipid accumulation in HepG2 cells}

To further investigate the possible mechanisms in the regulation of lipogenesis by FFAR1, an alternative and reliable model for studies of liver lipid metabolism,
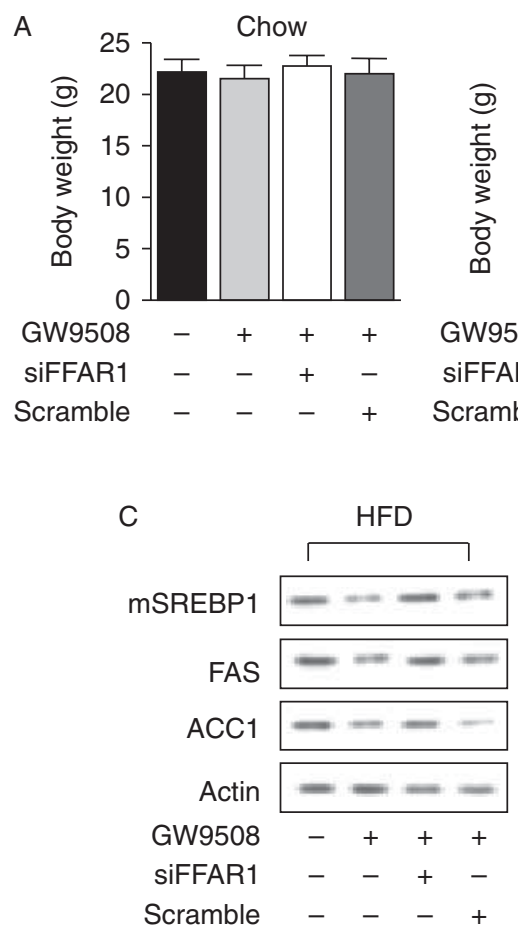
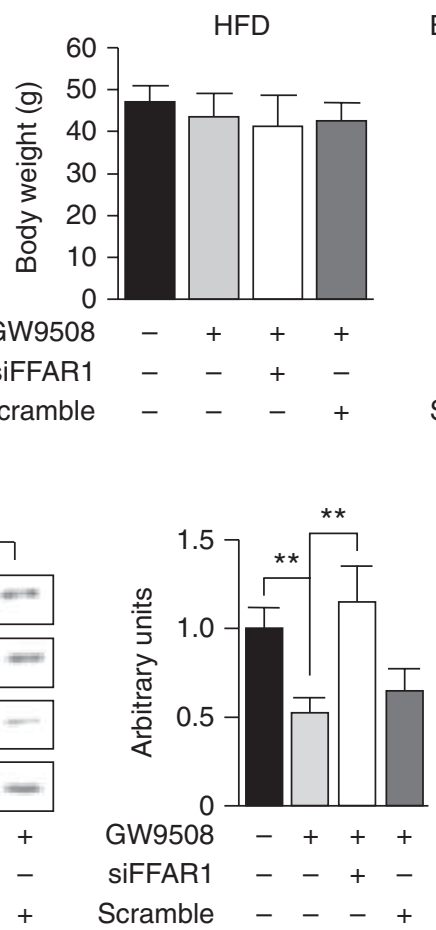

B
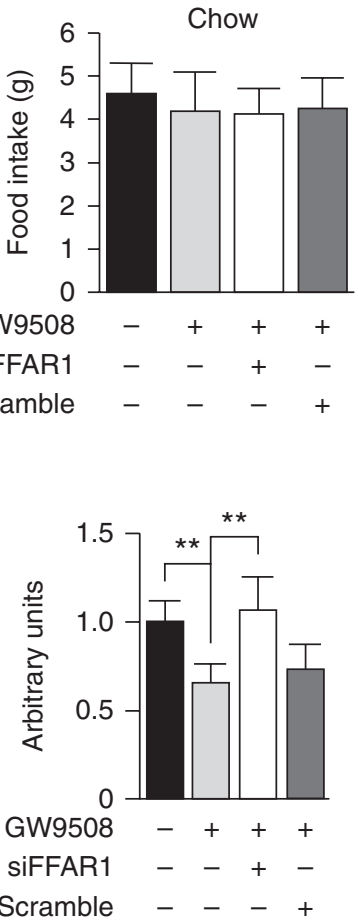
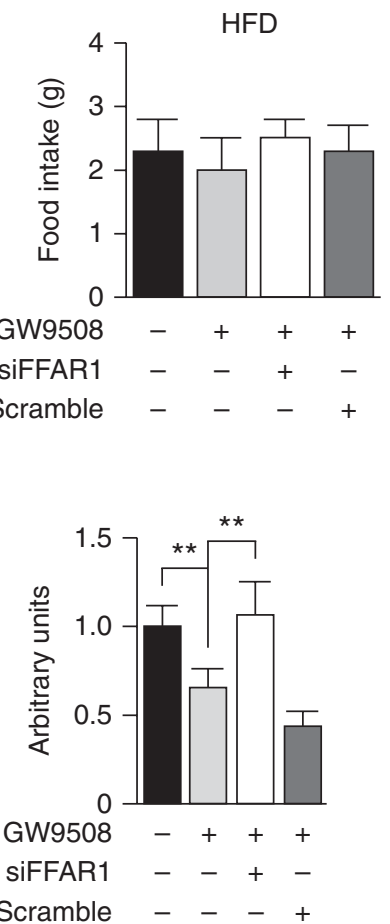

\section{Figure 3}

Knockdown of FFAR1 diminished the effect of GW9508 on expression of hepatic lipogenesis-related proteins without affecting body weight and food intake in animals. The body weight and food intake of each group of mice were also recorded (A and B). Expression of hepatic
๑) 2014 Society for Endocrinology Printed in Great Britain lipogenesis-related proteins in HFD mice treated with GW9508 were determined using western blotting analysis (C). The data were obtained from six to eight animals and expressed as mean \pm S.E.M. ${ }^{*} P<0.01$ as compared with the control group. 

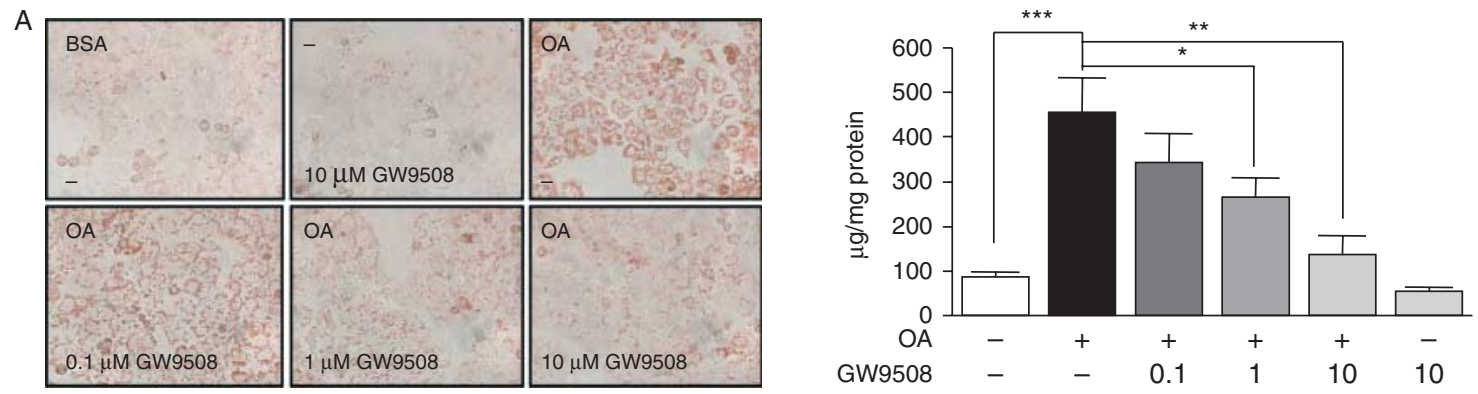

B

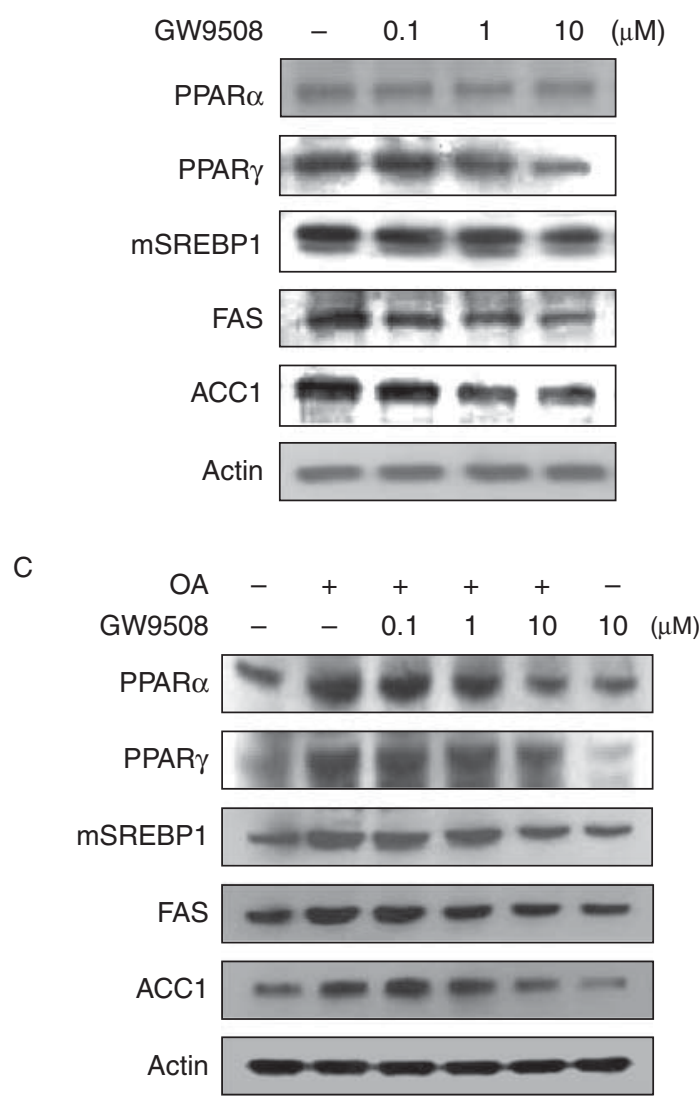

Figure 4

GW9508 decreased oleic acid (OA)-induced lipid accumulation in HepG2 cells. HepG2 cells were pretreated with indicated doses of GW9508 for $30 \mathrm{~min}$, and then treated with $0.5 \mathrm{mM}$ OA for $24 \mathrm{~h}$. The accumulation of lipid droplets was detected by Oil Red $\mathrm{O}$ staining and the intracellular triglyceride contents were also quantified (A). The levels of expression of lipogenesis-related proteins were determined by western blotting analysis
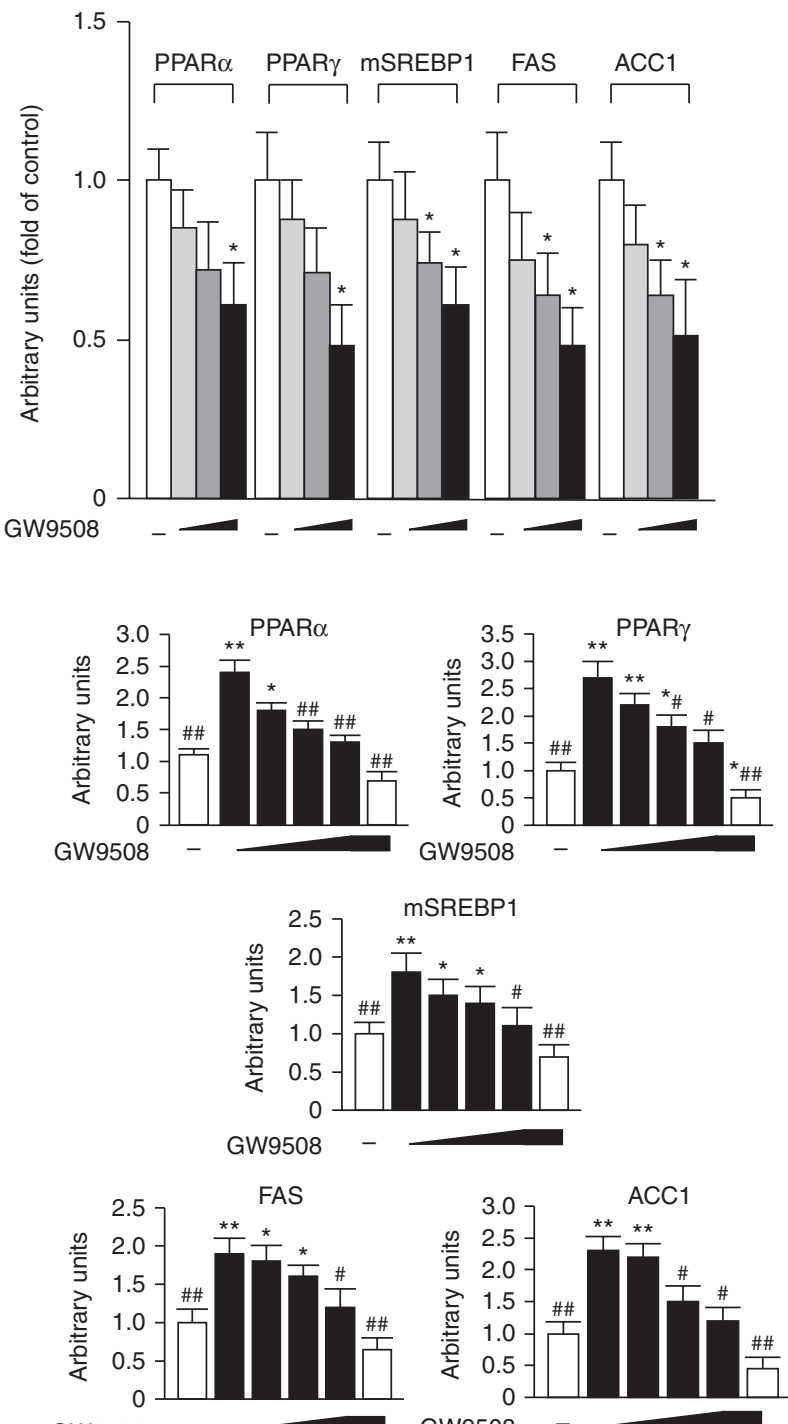

GW9508

GW9508

in GW9508-treated HepG2 cells (B) or combined with $0.5 \mathrm{mM}$ OA treatment for $24 \mathrm{~h}(\mathrm{C})$. The data were obtained from three individual experiments and expressed as mean \pm S.E.M. ${ }^{*} P<0.05, * * P<0.01$, and $* * * P<0.001$ as compared with the BSA control group. ${ }^{\#} P<0.05$ and ${ }^{\# \#} P<0.01$ as compared with the OA-treated group.

Published by Bioscientifica Ltd. 


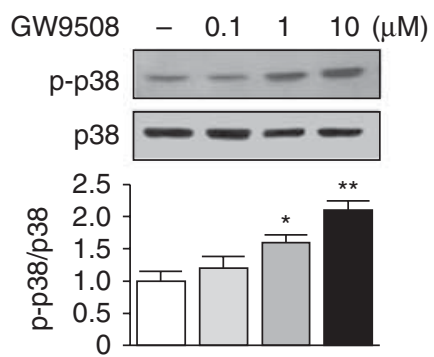

B
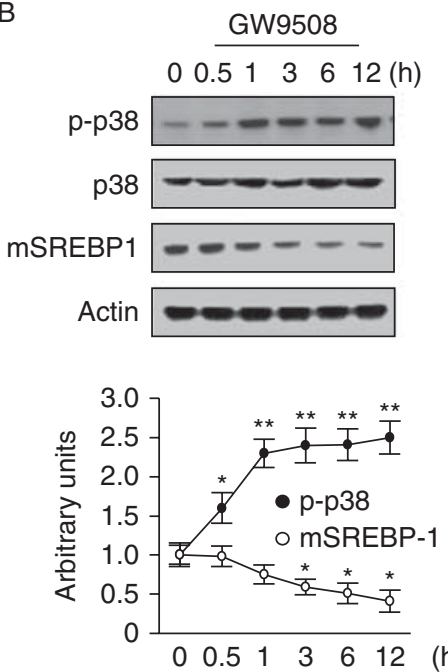

C
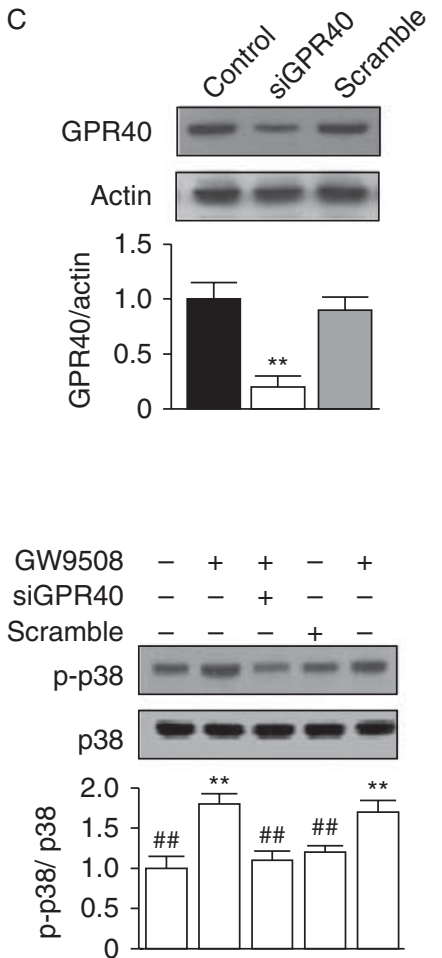

D
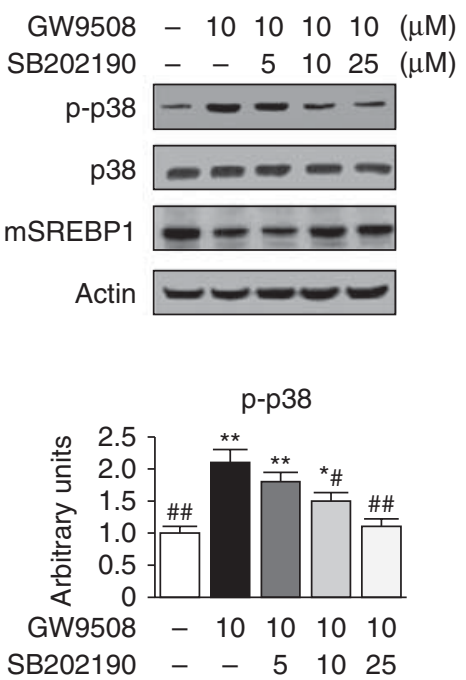

mSREBP1

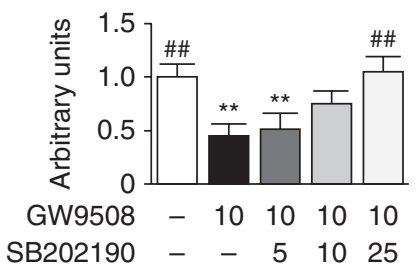

\section{Figure 5}

FFAR1 regulated SREBP1 activity through a p38-dependent pathway. HepG2 cells were treated with the indicated doses of GW9508 for $1 \mathrm{~h}$ to detect the phosphorylation of p38 (A). HepG2 cells were treated with $10 \mu \mathrm{M}$ GW9508 and then harvested at the indicated times for the detection of p38 phosphorylation and expression of mature SREBP1 (B). HepG2 cells were transfected with shRNA that targeted FFAR1, and then treated

the HepG2 hepatoma cell line was used (Wang et al. 1988). GW9508 significantly decreased the OA-induced lipid accumulation in HepG2 cells (Fig. 4A). In addition, GW9508 significantly decreased the expression of lipogenesis-related proteins (Fig. 4B). Moreover, OA-induced lipogenesis-related protein expressions were reversed by GW9508 (Fig. 4C).

\section{Activation of FFAR1 decreased OA-induced lipid accumulation through a p38-dependent pathway}

We then investigated the detailed mechanisms of action of FFAR1 in the regulation of lipogenesis-related protein expressions. Activation of FFAR1 by GW9508 activated p38 in a dose- (Fig. 5A) and time-dependent fashion (Fig. 5B). The phosphorylation of p38 was increased at with $10 \mu \mathrm{M}$ GW9508 for $1 \mathrm{~h}$ to detect the phosphorylation of p38 (C). Blockade of $\mathrm{p} 38$ phosphorylation by pretreatment with SB202190 inhibited GW9508-induced maturation of SREBP (D). Data were obtained from three individual experiments and expressed as mean \pm S.E.M. ${ }^{*} P<0.05$ and ${ }^{*} P<0.01$ as compared with the control group. ${ }^{\#} P<0.05$ and ${ }^{\# \#} P<0.01$ as compared with the GW9508-treated group.

$30 \mathrm{~min}$ after the treatment and reached a peak at $1 \mathrm{~h}$. FFAR1 knockdown diminished GW9508-induced p38 phosphorylation (Fig. 5C). In addition, treatment with a p38 inhibitor, SB202190, reversed the effect of GW9508 on the expression of mature SREBP1 (Fig. 5D). Moreover, the effects of GW9508 on insulin-induced lipogenesis were also evaluated. Insulin significantly increased the expression of SREBP1 (Fig. 6A), and pretreatment with GW9508 reversed the effect of insulin on SREBP1 expression (Fig. 6B). Knockdown of FFAR1 diminished the effect of GW9508 on insulininduced SREBP1 expression (Fig. 6C). Furthermore, confirming that activation of FFAR1 decreased OA-induced lipid accumulation through a p38-dependent pathway, we found that inhibition of p38 with SB202190 blocked the effect of GW9508 on OA-induced lipid accumulation in HepG2 cells (Fig. 6D).

Published by Bioscientifica Ltd 
A

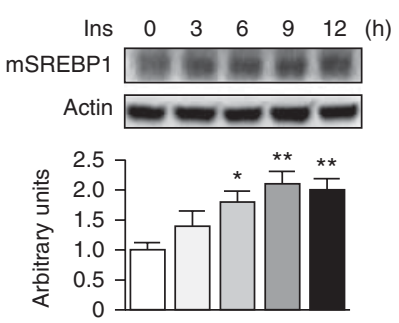

B

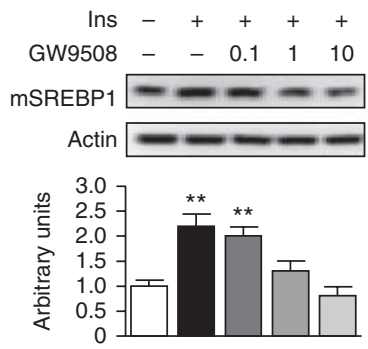

C

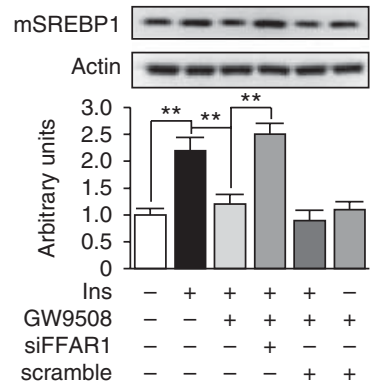

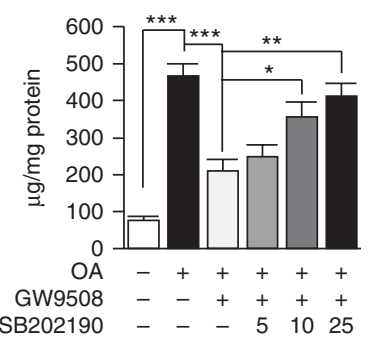

Figure 6

Knockdown of FFAR1 diminished the effect of GW9508 and insulin on hepatic lipogenesis. HepG2 cells were treated with $1 \mu \mathrm{M}$ insulin and then harvested at the indicated times for the detection of expression of mature SREBP1 (mSREBP1) (A). HepG2 cells were pretreated with $10 \mu \mathrm{M}$ GW9508 for $30 \mathrm{~min}$, and then treated with $1 \mu \mathrm{M}$ insulin for $24 \mathrm{~h}$ for the detection of the expression of mSREBP1 (B). Cells were tranfected with shRNA targeted to FFAR1, and pretreated with $10 \mu \mathrm{M} \mathrm{GW} 9508$ for $30 \mathrm{~min}$, and then treated

\section{FFAR1 activates p38 through a PLC-PKC-dependent pathway}

We further clarified the upstream signal transduction of FFAR1-induced p38 phosphorylation. Treatment with GW9508 immediately phosphorylated $\mathrm{PLC}_{\gamma}$ and

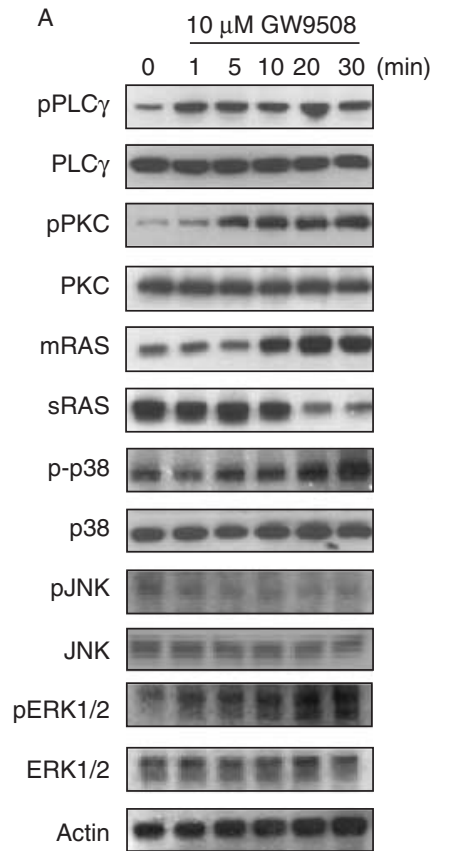

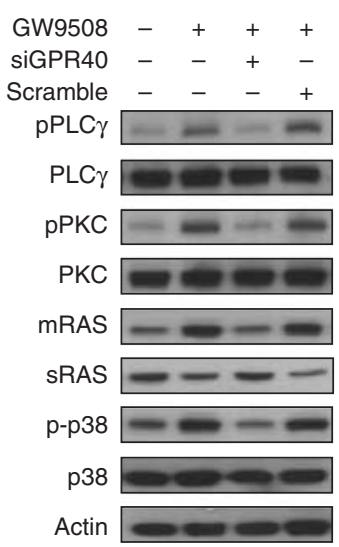

C GW9508 - + + + +

U73343 - -+--

U73122 - -++-

Chelethyrine $-{ }_{-}+{ }_{-}$

FTS $--\quad-\quad+$

pPLC $\gamma=-m-\infty$

PLC $\gamma=-----\infty$

pPKC $-\mathbf{m - m - m}$

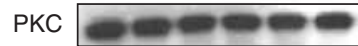

mRAS $-2-1-$

sRas $\mathbf{m - m - \infty}$

p-p38 $=---=$

p38 $-\mathrm{me-m-1}$

Actin $=\mathrm{ses}$

\section{Figure 7}

FFAR1 regulates $p 38$ activity through a PLC-PKC-RAS pathway. HepG2 cells were treated with $10 \mu \mathrm{M} \mathrm{GW} 9508$ and the protein samples were harvested at indicated times for the determination of protein expression (A). Cells were tranfected with shRNA targeted to FFAR1 and then the protein samples were harvested at $30 \mathrm{~min}$ for the determination of expression of proteins (B). HepG2 cells were pretreated with U73122 (1 $\mu \mathrm{M})$, U73343 $(1 \mu \mathrm{M})$, chelethyrine $(1 \mu \mathrm{M})$, or FTS $(10 \mu \mathrm{M})$ for $30 \mathrm{~min}$, and then treated with $10 \mu \mathrm{M} \mathrm{GW} 9508$ for 30 min for the determination of the expression of the indicated proteins expressions by western blotting analysis (C). mRAS, membrane RAS; cRAS, cytosolic RAS.

Published by Bioscientifica Ltd. 
and the translocation of RAS (Fig. 7B). In addition, pretreatment with PLC $\gamma$ (U73122), PKC (chelethyrine), and RAS (FTS) inhibitors reversed GW9508-induced p38 phosphorylation (Fig. 7C). Furthermore, pretreatment with U73122 inhibited PKC activation and chelethyrine reversed RAS translocation and FTS blocked p38 phosphorylation, indicating that FFAR1 activated p38 through a PLC-PKC-RAS pathway (Fig. 7C).

\section{Discussion}

The prevalence of diabetes is highly correlated with hepatic steatosis (Lewis \& Mohanty 2010). Recent studies had been focused on the therapeutic effects of FFAR1 agonists on diabetes (Hara et al. 2011). However, the role of FFAR1 in hepatic steatosis was still unknown. To the best of our knowledge, this is the first study to evaluate the effects of FFAR1 agonists on hepatic steatosis. We found that the FFAR1 agonist GW9508 significantly improved HFD-induced hepatic steatosis. In addition, hepatic FFAR1 knockdown diminished the effect of GW9508 on hepatic steatosis. Moreover, activation of FFAR1 decreased OA-induced lipid accumulation in HepG2 cells through a p38-dependent pathway.

The MAPK pathway is involved in the biological functions of FFAR1 (Seljeset \& Siehler 2012). FFAR1 activates ERK-MAPK pathway to control the plasticity of the $\beta$-cell mass and improve glycemic control (Zhang et al. 2007). FFAR1 activates ERK1/2 and p38 through a RAS-dependent mechanism to induce osteocyte apoptosis (Mieczkowska et al. 2012). In this study, we found that activation of p38 by FFAR1 is also involved in the regulation of hepatic lipogenesis.

The SREBP1 is a basic helix-loop-helix leucine-zippercontaining transcription factor, which is primarily involved in fatty acid synthesis by stimulating expression of the target genes, such as ACC and FAS. The function of SREBP1 is primarily regulated through protein cleavage and gene expression (Xiong et al. 2007). In addition, SREBP1 is regulated by the activity of p38 (Xiong et al. 2007). p38 plays an inhibitory role in hepatic lipogenesis and blockade of p38 leads to hypertriglyceridemia and fatty liver (Xiong et al. 2007). In this study, we found that FFAR1 regulated lipogenesis by decreasing SREBP1 through a p38-dependent pathway.

In addition to the role of $\mathrm{p} 38$ in the regulation of SREBP1, transcription of Srebp (Srebf1) in the liver is also regulated by insulin and glucagon. Insulin activates SREBP1 in liver, thereby increasing fatty acid and triglyceride synthesis (Owen et al. 2012). In our previous study, we found that administration of GW9508 to HFD mice decreases the serum insulin levels in a dosedependent manner. Results from a recent study have also indicated that although GW9508 stimulates a transient release of $\mathrm{Ca}^{2+}$ from intracellular $\mathrm{Ca}^{2+}$ stores via activation of PLC, GW9508 inhibits glucose-stimulated insulin secretion by activating KATP channels (Zhao et al. 2013). Thus, the decrease in insulin levels in response to GW9508 might be involved in the improvement of hepatic steatosis.

Although the FFAR1 agonists are applied in the treatment of metabolic diseases, and the therapeutic effects in diabetes have been demonstrated in humans (Kaku et al. 2013), the results from animal studies are still very different and obscure. Administration of FFAR1 agonists in diabetic animals improved glucose metabolism, and thus prevented the progression of diabetes (Ito et al. 2013, Ou et al. 2013, Tanaka et al. 2014). However, results from other studies have indicated that Ffar1-null mice did not show significant changes in glucose metabolism when fed either a chow diet or a HFD (Lan et al. 2008), and HFD-induced liver steatosis develops in both WT and Ffar1-null mice (Kebede et al. 2008). This discrepancy between studies may result from the different types of animal models. FFAR1 is an orphan receptor and the identity of its high-affinity endogenous ligand(s) is incompletely resolved, although FFAR1 binds and is activated by a variety of fatty acids. Thus, the physiological role of FFAR1 might not be significantly observable in an FFAR1-knockdown model. In addition, compensatory effects in gene-manipulated animal models might be a mechanism that leads to the lack of differences in glucose metabolism between WT and Ffar1null mice. In this study, we used a transient gene manipulation model in adult animals by means of lentiviral vector containing shFFAR1, which may decrease the effect of compensation as compared with embryonic FFAR1 knockdown. Also, as FFAR1 is an orphan receptor, direct activation by administration of FFAR1 agonists in animals may be useful for the investigation of FFAR1. However, the details of the mechanisms still need further study.

Taken together, the results presented in this work demonstrate that GW9508 may have a therapeutic effect in regulating hepatic lipid accumulation. Activation of FFAR1 by GW9508 increases the phosphorylation of p38 to regulate SREBP1 and further improves hepatic steatosis. Thus, the potential effects of GW9508 with regard to the treatment of hepatic steatosis in humans deserve further attention in future research.

Published by Bioscientifica Ltd. 


\section{Declaration of interest}

The authors declare that there is no conflict of interest that could be perceived as prejudicing the impartiality of the research reported.

\section{Funding}

The work was supported by Ministry of Science and Technology of Taiwan (102-2314-B-006-007, 102-2314-B-006-008, and 103-2314-B-006-027-MY2) and National Cheng Kung University Hospital (NCKUH-10104017)

\section{Author contribution statement}

$\mathrm{H}-\mathrm{Y} \mathrm{O}$ and $\mathrm{H}-\mathrm{T} \mathrm{W}$ initiated this study and organized all experiments. Y-C S, $\mathrm{H}-\mathrm{C} \mathrm{H}$, and F-H L drafted the manuscript and analyzed the data. Y-C Y, J-S W, and C-L W participated in the discussion of the experiments. C-J C designed the experiments and interpreted the results.

\section{References}

Christiansen E, Hansen SV, Urban C, Hudson BD, Wargent ET, Grundmann M, Jenkins L, Zaibi M, Stocker CJ, Ullrich S et al. 2013 Discovery of TUG-770: a highly potent free fatty acid receptor 1 (FFA1/GPR40) agonist for treatment of type 2 diabetes. ACS Medicinal Chemistry Letters 4 441-445. (doi:10.1021/ml4000673)

Feng DD, Luo Z, Roh SG, Hernandez M, Tawadros N, Keating DJ \& Chen C 2006 Reduction in voltage-gated $\mathrm{K}^{+}$currents in primary cultured rat pancreatic $\beta$-cells by linoleic acids. Endocrinology 147 674-682. (doi:10.1210/en.2005-0225)

Ferre P \& Foufelle F 2010 Hepatic steatosis: a role for de novo lipogenesis and the transcription factor SREBP-1c. Diabetes, Obesity \& Metabolism 12 83-92. (doi:10.1111/j.1463-1326.2010.01275.x)

Hara T, Hirasawa A, Ichimura A, Kimura I \& Tsujimoto G 2011 Free fatty acid receptors FFAR1 and GPR120 as novel therapeutic targets for metabolic disorders. Journal of Pharmaceutical Sciences 100 3594-3601. (doi:10.1002/jps.22639)

Ito R, Tsujihata Y, Matsuda-Nagasumi K, Mori I, Negoro N \& Takeuchi K 2013 TAK-875, a GPR40/FFAR1 agonist, in combination with metformin prevents progression of diabetes and $\beta$-cell dysfunction in Zucker diabetic fatty rats. British Journal of Pharmacology 170 568-580. (doi:10.1111/bph.12297)

Itoh Y, Kawamata Y, Harada M, Kobayashi M, Fujii R, Fukusumi S, Ogi K, Hosoya M, Tanaka Y, Uejima H et al. 2003 Free fatty acids regulate insulin secretion from pancreatic $\beta$ cells through GPR40. Nature $\mathbf{4 2 2}$ 173-176. (doi:10.1038/nature01478)

Kaku K, Araki T \& Yoshinaka R 2013 Randomized, double-blind, doseranging study of TAK-875, a novel GPR40 agonist, in Japanese patients with inadequately controlled type 2 diabetes. Diabetes Care 36 245-250. (doi:10.2337/dc12-0872)

Kebede M, Alquier T, Latour MG, Semache M, Tremblay C \& Poitout V 2008 The fatty acid receptor GPR40 plays a role in insulin secretion in vivo after high-fat feeding. Diabetes $\mathbf{5 7}$ 2432-2437. (doi:10.2337/db08-0553)

Lan H, Hoos LM, Liu L, Tetzloffl G, Hu WW, Abbondanzo SJ, Vassileva G, Gustafson EL, Hedrick JA \& Davis HR 2008 Lack of FFAR1/GPR40 does not protect mice from high-fat diet-induced metabolic disease. Diabetes 57 2999-3006. (doi:10.2337/db08-0596)
Lewis JR \& Mohanty SR 2010 Nonalcoholic fatty liver disease: a review and update. Digestive Diseases and Sciences 55 560-578. (doi:10.1007/ s10620-009-1081-0)

Mieczkowska A, Basle MF, Chappard D \& Mabilleau G 2012 Thiazolidinediones induce osteocyte apoptosis by a $\mathrm{G}$ protein-coupled receptor 40-dependent mechanism. Journal of Biological Chemistry 287 23517-23526. (doi:10.1074/jbc.M111.324814)

Ou HY, Wu HT, Hung HC, Yang YC, Wu JS \& Chang CJ 2013 Multiple mechanisms of GW-9508, a selective G protein-coupled receptor 40 agonist, in the regulation of glucose homeostasis and insulin sensitivity. American Journal of Physiology. Endocrinology and Metabolism 304 E668-E676. (doi:10.1152/ajpendo.00419.2012)

Owen JL, Zhang YY, Bae SH, Farooqi MS, Liang GS, Hammer RE, Goldstein JL \& Brown MS 2012 Insulin stimulation of SREBP-1C processing in transgenic rat hepatocytes requires p70 S6-kinase. PNAS 109 16184-16189. (doi:10.1073/pnas.1213343109)

Seljeset S \& Siehler S 2012 Receptor-specific regulation of ERK1/2 activation by members of the "free fatty acid receptor" family. Journal of Receptors and Signal Transduction 32 196-201. (doi:10.3109/10799893.2012. 692118)

Spector AA 1975 Fatty-acid binding to plasma albumin. Journal of Lipid Research 16 165-179.

Suh HN, Huong HT, Song CH, Lee JH \& Han HJ 2008 Linoleic acid stimulates gluconeogenesis via $\mathrm{Ca}^{2+} / \mathrm{PLC}, \mathrm{CPLA}_{2}$, and PPAR pathways through GPR40 in primary cultured chicken hepatocytes. American Journal of Physiology. Cell Physiology 295 C1518-C1527. (doi:10.1152/ ajpcell.00368.2008)

Tanaka H, Yoshida S, Minoura H, Negoro K, Shimaya A, Shimokawa T \& Shibasaki M 2014 Novel GPR40 agonist AS2575959 exhibits glucose metabolism improvement and synergistic effect with sitagliptin on insulin and incretin secretion. Life Sciences 94 115-121. (doi:10.1016/ j.lfs.2013.11.010)

Wang SR, Pessah M, Infante J, Catala D, Salvat C \& Infante R 1988 Lipid and lipoprotein metabolism in hep-G2 cells. Biochimica et Biophysica Acta 961 351-363. (doi:10.1016/0005-2760(88)90082-3)

Wang CR, Shiau AL, Chen SY, Cheng ZS, Li YT, Lee CH, Yo YT, Lo CW, Lin YS, Juan HY et al. 2010 Intra-articular lentivirus-mediated delivery of galectin-3 shRNA and galectin-1 gene ameliorates collagen-induced arthritis. Gene Therapy 17 1225-1233. (doi:10.1038/gt.2010.78)

Wu HT, Chen W, Cheng KC, Ku PM, Yeh CH \& Cheng JT 2012 Oleic acid activates peroxisome proliferator-activated receptor $\delta$ to compensate insulin resistance in steatotic cells. Journal of Nutritional Biochemistry $\mathbf{2 3}$ 1264-1270. (doi:10.1016/j.jnutbio.2011.07.006)

Wu HT, Lu FH, Ou HY, Su YC, Hung HC, Wu JS, Yang YC, Wu CL \& Chang CJ 2013 The role of Hepassocin in the development of non-alcoholic fatty liver disease. Journal of Hepatology 59 1065-1072. (doi:10.1016/ j.jhep.2013.06.004)

Xiong Y, Collins QF, An J, Lupo E, Liu HY, Liu DL, Robidoux J, Liu ZQ \& Cao WH 2007 p38 mitogen-activated protein kinase plays an inhibitory role in hepatic lipogenesis. Journal of Biological Chemistry $\mathbf{2 8 2}$ 4975-4982. (doi:10.1074/jbc.M606742200)

Zhang Y, Xu M, Zhang S, Yan L, Yang C, Lu W, Li Y \& Cheng H 2007 The role of $\mathrm{G}$ protein-coupled receptor 40 in lipoapoptosis in mouse $\beta$-cell line NIT-1. Journal of Molecular Endocrinology 38 651-661. (doi:10.1677/ JME-06-0048)

Zhao YF, Wang L, Zha D, Qiao L, Lu L, Yu J, Qu P, Sun Q, Qiu J \& Chen C 2013 GW9508 inhibits insulin secretion by activating ATP-sensitive potassium channels in rat pancreatic $\beta$-cells. Journal of Molecular Endocrinology 51 69-77. (doi:10.1530/JME-13-0019)

Received in final form 1 July 2014

Accepted 8 July 2014

Accepted Preprint published online 8 July 2014 http://jme.endocrinology-journals.org DOI: 10.1530/JME-14-0003
() 2014 Society for Endocrinology Printed in Great Britain 